\title{
Lacosamide for the prevention of partial onset seizures in epileptic adults
}

\author{
This article was published in the following Dove Press journal: \\ Neuropsychiatric Disease and Treatment \\ 23 July 2010 \\ Number of times this article has been viewed
}

\author{
Anna Kelemen' \\ Péter Halász ${ }^{2}$ \\ 'National Institute of Neurosciences, \\ Epilepsy Center, Budapest, Hungary; \\ ${ }^{2}$ Faculty of Information Technology, \\ Pázmány Péter Catholic University, \\ Budapest, Hungary
}

\begin{abstract}
Lacosamide is a newly registered antiepileptic drug with dual mechanisms of action. It selectively enhances slow inactivation of voltage-gated sodium channels, resulting in stabilization of hyperexcitable neuronal membranes and inhibition of repetitive neuronal firing. It also binds to a collapsing-response mediator protein-2, CRMP2. Lacosamide has a favorable pharmacokinetic profile; is rapidly and completely absorbed, has a relatively long elimination half-life of 13 hours which allows twice-daily administration, linear pharmacokinetics, and has low potential for drug interactions and renal elimination. Both oral and intravenous formulations of lacosamide are being developed. In placebo-controlled clinical trials, lacosamide was effective in seizure reduction as adjunctive therapy in patients with uncontrolled partial-onset seizures. Lacosamide was generally well tolerated. The most frequently reported adverse events in placebo-controlled trials were dizziness, headache, nausea, and diplopia. Intravenous lacosamide has a comparably good safety profile.
\end{abstract}

Keywords: lacosamide, epilepsy, partial onset seizures

\section{Introduction}

Epilepsy is one of the most common neurological disorders, with an annual incidence in developed countries of 24-53 cases per 100,000 person-years, and with an ageadjusted incidence of all unprovoked seizures of 61 per 100,000 person-years. ${ }^{1}$ The age-adjusted prevalence of epilepsy per 1,000 population varies widely; most studies show a range between $4-8 .^{2}$ Three percent of the general population have had a seizure at some point in their lives. The prevalence of individuals receiving treatment for epilepsy is about 1 in $200 .^{3}$

Epilepsy is characterized by the occurrence of at least two unprovoked episodes of periodic disturbance in neurological function, often with altered consciousness, due to an abnormal excessive electrical discharge within the brain. In generalized epilepsy syndromes, the predominant type of seizures involves early both cerebral hemispheres. By contrast, seizures in partial epilepsy syndromes involve a localized portion of one hemisphere of the brain at their onset, although they can spread to involve the entire brain, a process known as secondary generalization.

Seizures can be classified as being generalized or partial. Generalized seizures may be tonic/clonic, isolated tonic or clonic, myoclonic, or absence seizures. Partial seizures may be divided into simple partial (motor or sensory) with retained awareness or complex partial with impaired awareness. Partial seizures may progress into generalized seizures. Partial seizures are the most frequent and most difficult to control. ${ }^{4}$ submit your manuscript $\mid$ www.dovepress.com

Dovepress

7967 


\section{Lacosamide, a new antiepileptic drug Introduction}

Lacosamide is a new antiepileptic drug recently approved by the European Medicines Agency (EMEA) and by the US Food and Drug Administration (FDA) in 2008 as adjunctive therapy for partial-onset seizures with (EC) or without (EMEA, FDA) secondary generalization in patients with epilepsy aged above 16 years (EC) and 17 years (FDA). ${ }^{5,6}$ It is available for oral and intravenous administration. Traditional antiepileptic drugs (AEDs) are effective in suppressing seizure activity; however, their utility is limited by significant side effects and by their propensity for drug-drug interactions. In the past 15 years, multiple second-generation AEDs have been introduced. Many of them have better tolerability profiles and a lower drug interaction potential. Nevertheless, about a third of patients with epilepsy are refractory to available treatments, and a significant need for novel drugs remains. ${ }^{7,8}$

\section{Pharmacodynamics}

Lacosamide, (R)-2-acetamido-N-benzyl-3-methoxypropionamide, is a new compound specifically synthesized as an anticonvulsive drug candidate. ${ }^{9}$ In the mid-1980s, it was reported that N-acetyl-D,L-alanine benzylamide was effective in animal models of epilepsy. ${ }^{10}$ Systematic evaluation of more than 100 derivatives of this compound in animal models led to the identification of lacosamide. ${ }^{11}$

Lacosamide seems to have a novel dual mode of action. It selectively enhances slow inactivation of voltage-gated sodium channels, resulting in stabilization of hyperexcitable neuronal membranes and inhibition of repetitive neuronal firing without exhibiting effects on physiological neuronal excitability (Figure 1). ${ }^{12}$ Lacosamide also binds to collapsing-response mediator protein-2 (CRMP2), a phosphoprotein that is expressed mainly in the nervous system. CRMP-2 is involved in neuronal differentiation and network building and control of axonal outgrowth and probably also epileptogenesis. ${ }^{13}$ Further research is needed to determine whether the interaction between lacosamide and CRMP2 leads to symptomatic or disease-modifying effects. ${ }^{14}$

In preclinical studies, lacosamide was evaluated in the antiepileptic drug (AED) development program of the Epilepsy Branch of the National Institutes of Health (NIH) and was found to be more potent and effective in several animal models compared to other AEDs. Lacosamide inhibited N-methyl-D-aspartic acid (NMDA)-induced seizures and showed full efficacy in the homocysteine model of epilepsy.
It was also effective against sound-induced seizures in the genetically susceptible Frings mouse, against maximal electroshock test (MES)-induced seizures, in the rat hippocampal kindling model of partial seizures and in the $6 \mathrm{~Hz}$ model of psychomotor seizures in mice. Lacosamide failed to show efficacy in some other animal models such as picrotoxine, bicuculline, and partly in pentylenetetrazol. ${ }^{6}$

\section{Pharmacokinetics}

Lacosamide is absorbed rapidly and completely from the gastrointestinal tract after oral administration with negligible first-pass effect, and has a high oral bioavailability (approximately $100 \%$ ), which is not affected by food. ${ }^{15}$ Peak plasma concentrations are achieved between 0.5 and 4 hours following a single dose. Lacosamide has a linear pharmacokinetics; plasma concentrations increase proportionally with dose in the therapeutic range (50-300 mg intravenous and 100-800 mg oral), with low intra- and interpatient variability. ${ }^{14}$

The elimination half-life of lacosamide is 13 hours and is stable in repeated administration. Lacosamide and its metabolites are primarily eliminated renally. The major metabolic pathway is demethylation to an O-desmethyl metabolite, which has not shown any pharmacological activity. Lacosamide has a low pharmacokinetic interaction potential. Lacosamide does not inhibit or induce the cytochrome P450 enzyme family at therapeutic concentrations. ${ }^{14}$

It has low protein binding $(<15 \%) \cdot{ }^{16}$ Results from clinical efficacy and safety trials showed that lacosamide does not affect the plasma levels of carbamazepine, valproic acid, lamotrigine, levetiracetam, oxcarbazepine, or phenytoin to a relevant extent, although the levels of the effective metabolite of oxcarbazepine, the monohydroxy-derivative was reduced by $10 \%-15 \% .{ }^{17}$

Similarly, these drugs do not affect the pharmacokinetics of lacosamide in a clinically relevant manner although enzyme-inducing drugs reduced the overall systemic exposure to lacosamide in the phase III study patients. Lacosamide does not affect the levels of metformin, digoxin, or oral contraceptives. ${ }^{6}$

Lacosamide solution for infusion $(10 \mathrm{mg} / \mathrm{mL})$ is isotonic, stable at room temperature, and does not require dilution prior to intravenous administration. Intravenous infusion of lacosamide at a dose of $200 \mathrm{mg}$ over 30 or 60 minutes has shown bioequivalence (maximum plasma concentration, area under the curve) to oral lacosamide at the same dose. ${ }^{18}$

\section{Efficacy}

Three multicenter, randomized, double-blind, phase II/III, placebo-controlled trials evaluated the efficacy and safety of 
oral lacosamide when administered concomitantly with up to three AEDs in patients with uncontrolled partial-onset seizures, with or without secondary generalization. These trials were similar in design but different in doses investigated and ages of the patients enrolled; the doses were $200 \mathrm{mg}, 400 \mathrm{mg}$, and $600 \mathrm{mg}$ in the SP667 trial, $200 \mathrm{mg}$ and $400 \mathrm{mg}$ in the SP755 trial, and $400 \mathrm{mg}$ and $600 \mathrm{mg}$ in the SP754 trial. ${ }^{19-21}$

In all trials, 1,308 patients with a history of an average of 23 years of partial-onset seizures were involved. Enrolled patients reported an average of at least four partial-onset seizures per 28 days, with seizure-free periods no longer than 21 days, during the eight-week period before enrollment as well as during the eight-week baseline period. In these trials, patients were randomized to receive adjunctive lacosamide or placebo. During the titration period of each trial, the lacosamide dose was increased to the randomized dose with forced weekly titrations of $100 \mathrm{mg} /$ day (50 mg twice daily). This titration period was followed by a 12 -week maintenance period. Efficacy was evaluated on an intent-to-treat basis with analysis of seizure frequency data, comparing maintenance data with baseline data.

In the first trial, 418 patients taking one or two concomitant AEDs were randomized to placebo or lacosamide $200 \mathrm{mg}, 400 \mathrm{mg}$, or $600 \mathrm{mg} .{ }^{19}$ Statistically significant reductions in seizure frequency per 28 days were observed in the $400 \mathrm{mg} /$ day $(P=0.002)$ and $600 \mathrm{mg} /$ day $(P=0.008)$ lacosamide treatment groups, but not in the $200 \mathrm{mg}$ /day group, compared with placebo. Statistically significant differences in responder rate (number of patients with $>50 \%$ reduction in seizure frequency per 28 days), was significant over placebo (for which the responder rate was $22 \%$ ) for lacosamide $400 \mathrm{mg}(41 \%)(P=0.004)$ and $600 \mathrm{mg} / \mathrm{day}(38 \%)(P=0.014)$, but was not significant in the $200 \mathrm{mg} /$ day arm. ${ }^{19}$

In the second trial involving 485 patients taking up to three concomitant AEDs and randomized to placebo or lacosamide $200 \mathrm{mg}$ or $400 \mathrm{mg}$. Statistically significant reductions in seizure frequency per 28 days were observed in the $200 \mathrm{mg}$ /day

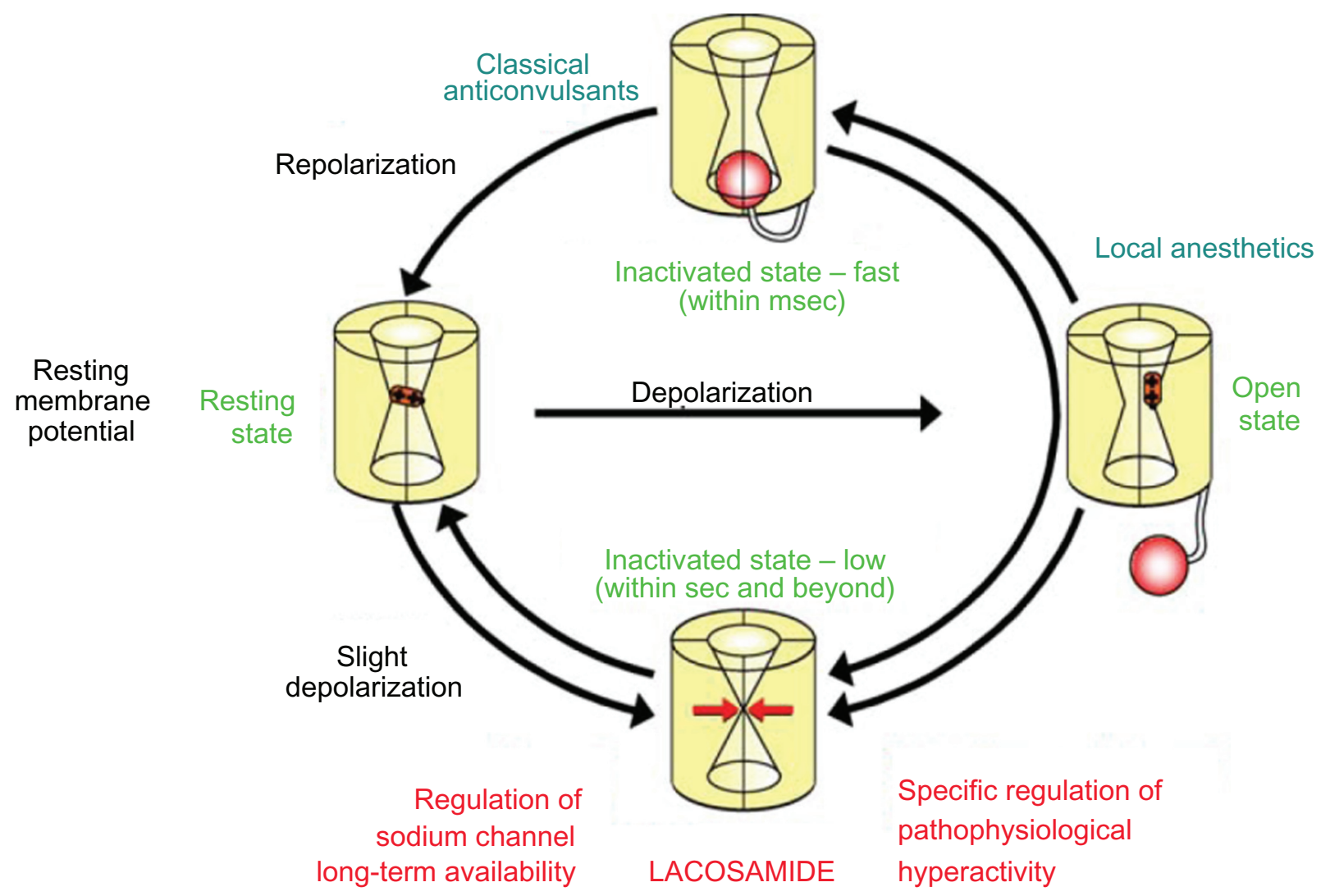

Figure I Working modes of voltage-gated sodium channels. At the resting potential, sodium channels are closed and can be opened by depolarization of the membrane potential which allow the flux of sodium ions into the cell. The channels close within a few milliseconds from inside of the neuron and go into a fast inactivated state from which they cannot be reactivated. When the membrane potential returns to baseline, the sodium channel goes back to its resting state. Under conditions of slight prolonged depolarization and repetitive neuronal activity, the sodium channel can go into a slow inactivated state by closing the pore from inside. This process happens on a second-to-minute time scale. Drugs can either block the open channel (eg, local anesthetics), or enhance fast inactivation (classical anticonvulsants), or enhance slow inactivation (lacosamide). Copyright () 2007. John Wiley and Sons. Reproduced with permission from Beyreuther B, Freitag J, Heers C, Krebsfänger N, Scharfenecker U, Stöhr T. Lacosamide: a review of preclinical properties. CNS Drug Rev. 2007; 13:2।-42. 
$(P=0.022)$ and $400 \mathrm{mg} /$ day $(P=0.033)$ lacosamide treatment groups compared with placebo. Statistically significant differences in responder rate (number of patients with $50 \%$ reduction in seizure frequency per 28 days) were observed for patients treated with lacosamide $400 \mathrm{mg} /$ day $(41 \%)(P=0.006)$, responder rate was significantly greater for lacosamide $400 \mathrm{mg}$ than for placebo $(26 \%){ }^{20}$

In the third trial, 405 patients with partial-onset seizures, taking up to three concomitant AEDs were randomized to one of three treatment groups: placebo, lacosamide $400 \mathrm{mg} /$ day, or lacosamide $600 \mathrm{mg} /$ day. Fifty percent responder rates on both dosages tested (400 mg and $600 \mathrm{mg}$ ) were significantly superior to placebo $(38 \%$ and $41 \%$ versus $18 \%$, respectively). ${ }^{21,22}$

Overall lacosamide showed a clear efficacy measured in the proportion of patients with a more than $50 \%$ reduction in seizure frequency: $40 \%$ for lacosamide $400 \mathrm{mg}$ per day, $34 \%$ for lacosamide $200 \mathrm{mg}$, and $23 \%$ for placebo (Figure 2). ${ }^{23}$ A statistically significant reduction in 28-day seizure frequency (baseline to maintenance phase) compared with the placebo group was observed with lacosamide $200 \mathrm{mg}$ per day in the second trial, $400 \mathrm{mg}$ per day in all three studies, and $600 \mathrm{mg}$ per day in both studies with this dosage (Figure 3). ${ }^{23}$ The efficacy of lacosamide $600 \mathrm{mg}$ per day was similar to that of lacosamide $400 \mathrm{mg}$ per day, but patients were less likely to tolerate this dose because of central nervous system- and gastrointestinal-related adverse effects, therefore the maximum recommended dose is $400 \mathrm{mg}$ per day. ${ }^{24}$ In terms of seizure freedom, which is most relevant for quality of life, results were $2.5 \%-4.6 \%$ of patients randomized to receive $400 \mathrm{mg}$ per day of lacosamide in different trials rendered seizure free. More than $90 \%$ of eligible patients who completed a placebo-controlled trial chose to receive lacosamide treatment in an open-label study.

The retention rate was not one of the prespecified outcome parameters. However, the retention rate is an important variable because it combines aspects of efficacy as well as of tolerability. Eighty-six of 97 patients randomized to placebo (89\%), 85 of 107 patients randomized to lacosamide $200 \mathrm{mg} /$ day (79\%), 80 of 108 patients randomized to lacosamide $400 \mathrm{mg} /$ day

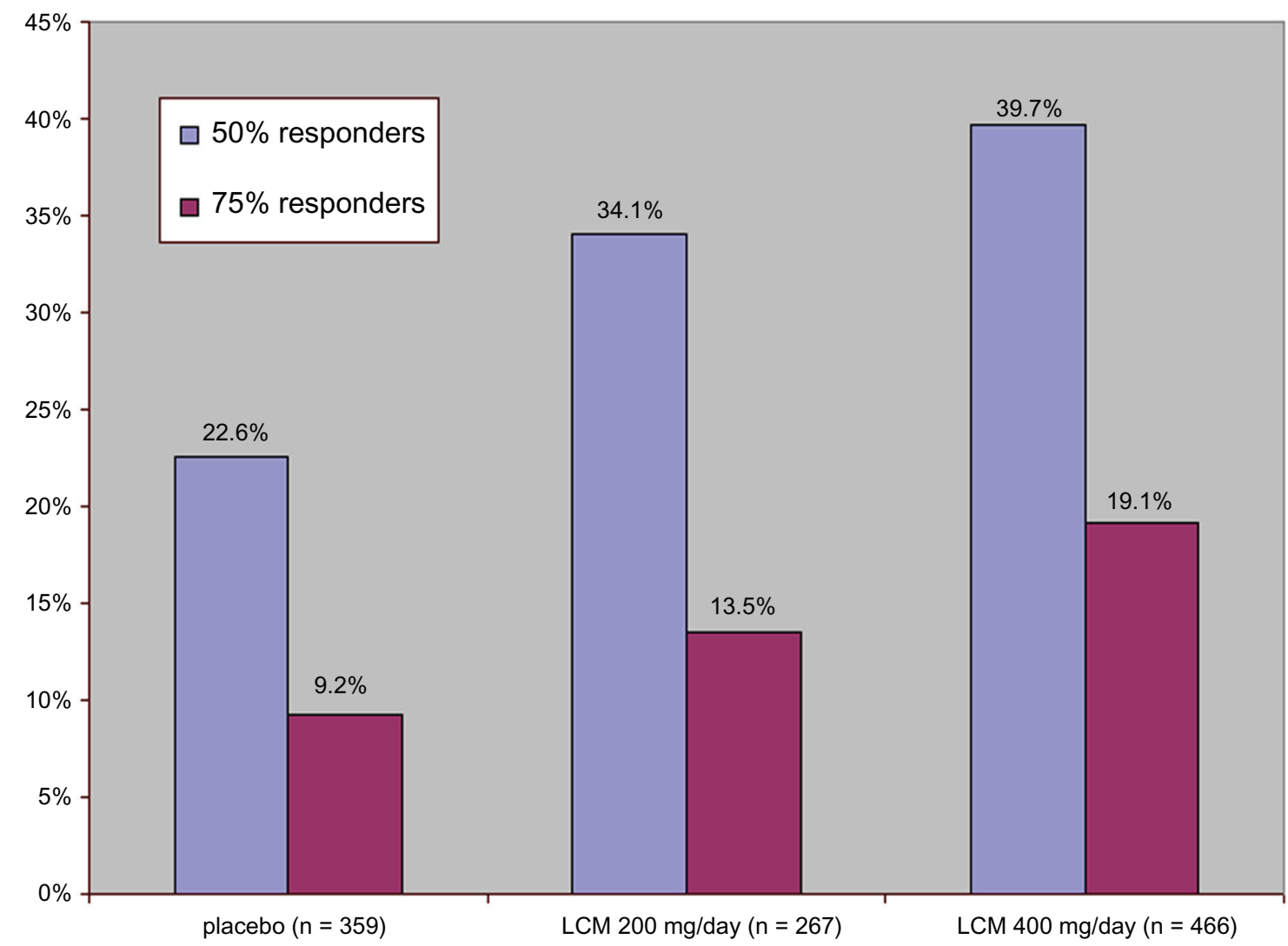

Figure 2 Percentage of patients with at least $50 \%$ or $75 \%$ reduction in seizure frequency from baseline period to the maintenance period. Pooled efficacy data of SP667, SP754, and SP755 trials.

Notes: $* P<0.05$, $* * P<0.001$ vs placebo. Inferential statistics for the $75 \%$ responders are not available. Copyright (C) 2009 Expert Reviews Ltd. Reproduced with permission from Beydoun A, D'Souza J, Hebert D, Doty P. Lacosamide: pharmacology, mechanisms of action and pooled efficacy and safety data in partial-onset seizures. Expert Rev Neurother. 2009;9(I):33-42.

Abbreviation: LCM, lacosamide. 


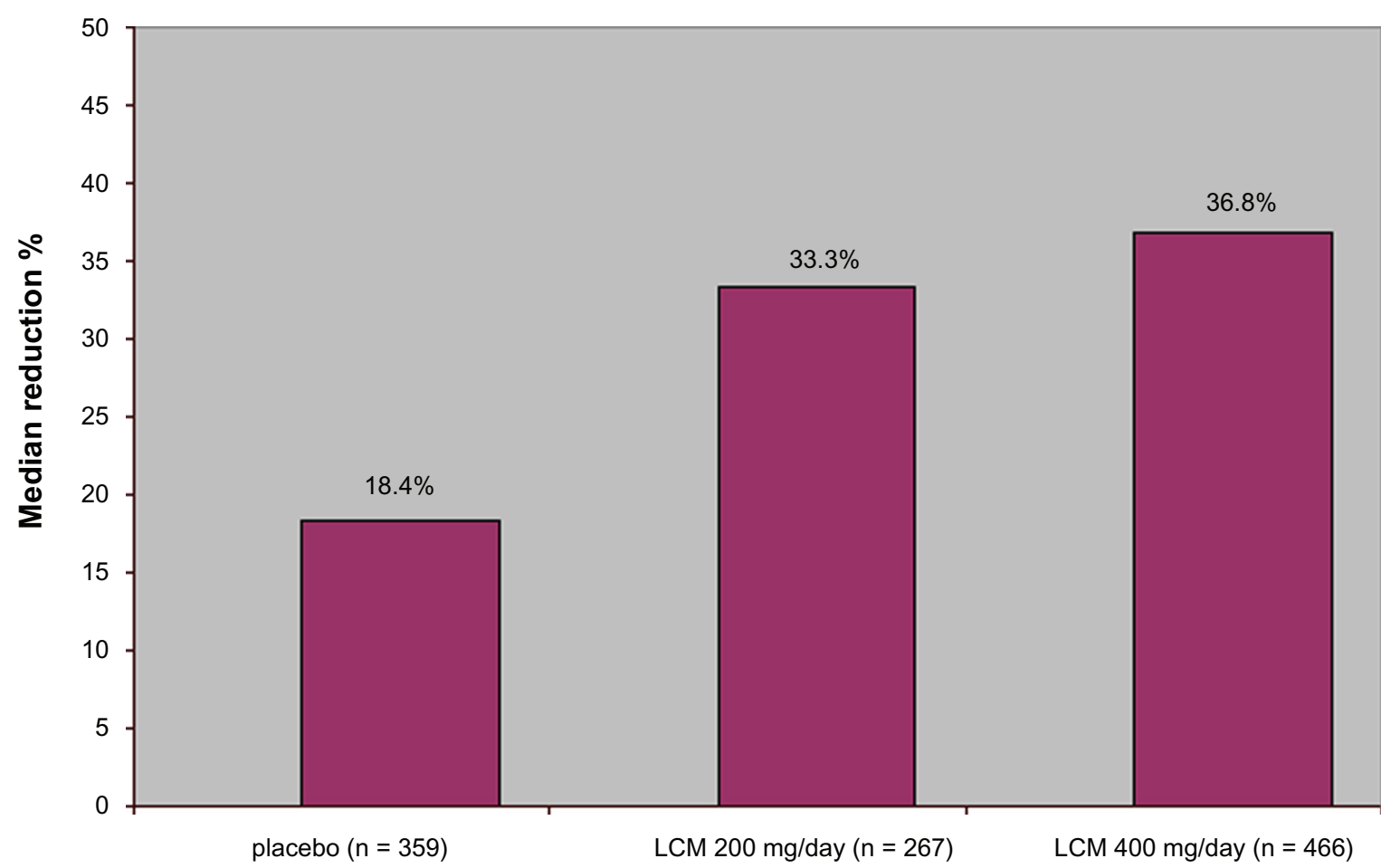

Figure 3 Median percentage reduction in seizure frequency per 28 days from the baseline period to the maintenance period. Pooled efficacy data of SP667, SP754, and SP755 trials.

Notes: ${ }^{*} P<0.05,{ }^{* * P}<0.001$ vs placebo. Inferential statistics for the $75 \%$ responders are not available. Copyright (C) 2009. Reproduced with permission from Beydoun A, D'Souza J, Hebert D, Doty P. Lacosamide: pharmacology, mechanisms of action and pooled efficacy and safety data in partial-onset seizures. Expert Rev Neurother. $2009 ; 9$ (I):33-42.

Abbreviation: LCM, lacosamide.

(74\%), and 61 of 106 patients randomized to lacosamide $600 \mathrm{mg} /$ day $(57 \%)$ completed the trial..$^{25}$

\section{Safety}

In the three completed placebo-controlled trials, the most frequently reported treatment-emergent adverse events were in the nervous and gastrointestinal systems, such as dizziness, headache, and nausea. ${ }^{24}$ Most often these events were mild or moderate in intensity. The incidence of dizziness and nausea appeared to be dose-related, increasing linearly with increasing doses of lacosamide (Table 1). ${ }^{23}$ Discontinuation rates due to adverse events also appeared to be related to dose. As a dose of $600 \mathrm{mg} /$ day is not more effective and carries the risk of more frequent side effects, the highest recommended dose is $400 \mathrm{mg}$ daily. Generally, no clinically important changes were observed in body weight, laboratory, vital signs or electrocardiography (ECG) variables. There was, however, a small dose-related increase in PR interval (mean change from baseline at the end of maintenance of $4.2 \mathrm{~ms}$ to $4.6 \mathrm{~ms}$ for the $400 \mathrm{mg}$ /day dose) observed on the ECG. ${ }^{19,20}$

In the open-label extension trials, the types of adverse events that emerged with chronic therapy were generally similar to those observed in the placebo-controlled trials. ${ }^{24}$
Serious adverse events occurred in $3 \%-7 \%$ in the placebo group, $3 \%-9 \%$ in the lacosamide $200 \mathrm{mg} /$ day group, $5 \%-10 \%$ in the lacosamide $400 \mathrm{mg} /$ day group, and $3 \%-10 \%$ in the lacosamide $600 \mathrm{mg}$ /day group. The serious adverse events seen in more than one patient were: worsening of seizures, psychotic disorders, dizziness, vomiting, accident, and nystagmus..$^{19.20}$

\section{Intravenous lacosamide}

Two intravenous lacosamide trials were carried out. A multicenter, double-blind, double-dummy, inpatient, randomized controlled trial evaluated the safety and tolerability of intravenous lacosamide as replacement for oral lacosamide for up to two days. Sixty patients from an ongoing openlabel extension trial of oral lacosamide were randomized in a 2:1 ratio to either intravenous lacosamide and oral placebo or intravenous placebo and oral lacosamide. Half of the enrolled patients received 60-minute infusions, and the other half received 30-minute infusions. The infusion dosage for each patient was (100-300 mg twice daily) was identical on a milligram basis to the oral dose of lacosamide that the patient had been receiving in the open-label extension trial. After completing this trial, patients resumed participation in the open-label extension trial with oral lacosamide. ${ }^{26}$ 
Table I Incidence of most common treatment emergent adverse effects leading to early discontinuation in the lacosamide $200 \mathrm{mg} / \mathrm{day}$ and $400 \mathrm{mg} /$ day groups during the treatment period

\begin{tabular}{llll}
\hline Kind of side effect & Placebo \% $(\mathbf{n}=\mathbf{3 6 4})$ & LCM $\mathbf{2 0 0} \mathbf{~ m g / d a y ~ \% ~}(\mathbf{n}=\mathbf{2 7 0})$ & LCM 400 $\mathbf{~ g / d a y ~ \% ~}(\mathbf{n}=\mathbf{4 7}$ I $)$ \\
\hline Dizziness & 0.3 & 0.4 & 4.2 \\
Vomiting & 0.3 & 0.4 & 2.3 \\
Diplopia & 0.3 & 1.5 & 2.1 \\
\hline
\end{tabular}

Notes: Pooled data of SP667,Sp754, and SP 755 trials. Copyright @ 2009. Reproduced with permission from Beydoun A, D’Souza J, Hebert D, Doty P. Lacosamide: pharmacology, mechanisms of action and pooled efficacy and safety data in partial-onset seizures. Expert Rev Neurother. 2009;9(1):33-42.

Abbreviation: LCM, lacosamide.

A recently completed, multicenter, open-label, inpatient trial evaluated the safety and tolerability of intravenous lacosamide (100-400 mg twice daily) when administered as short-term replacement for oral lacosamide for up to five days. ${ }^{27}$ For the 160 patients enrolled from the ongoing open-label extension trials, intravenous lacosamide was administered at infusion durations of 30 minutes $(n=40)$, 15 minutes $(\mathrm{n}=100)$, or 10 minutes $(\mathrm{n}=20)$. Across all infusion durations, most patients received twice-daily doses of lacosamide in the $200-400 \mathrm{mg}$ range. Approximately half of the patients were exposed to four or five days of treatment with twice-daily infusions of intravenous lacosamide. Intravenous lacosamide at doses of 100-400 mg twice daily and infusion durations of $60,30,15$, and 10 minutes demonstrated a comparable safety profile to oral lacosamide. Infusion site-related adverse events were infrequent and did not result in discontinuation from the trial. A case of a refractory convulsive status epilepticus successfully controlled with lacosamide was reported..$^{28}$

Bioequivalence between the oral tablet and the oral syrup of lacosamide has been established. ${ }^{29}$

Postmarketing data showed comparable efficacy to clinical trials (32\% reported a greater than $50 \%$ reduction in seizure frequency) and more side effects (52\%), mostly dizziness, fatigue, nausea, gait instability during the titration phase and $10 \%$ weight loss. ${ }^{30}$

\section{Conclusion}

Lacosamide is a useful antiepileptic drug for difficult-totreat partial-onset seizures. Its new mechanism of action might indicate a possibility of the drug to be advantageous in combination therapy. Its advantages seem to be good pharmacokinetics, a low potential for drug-drug interactions including oral contraceptives and anticoagulants, which make the drug appropriate for broad usage. The favorable adverse effect profile lacking sedative effect, the availability of an intravenous formulation that may be used for replacement therapy in patients temporarily unable to take oral medication, and the antineuralgic effect, useful in patients having neuropathy as co-morbidity, are the main features of the drug. Disadvantages include the need for slow-dose titration and an apparently narrow effective dose range and of potential ECG changes suggesting that caution is needed before using this drug in patients with pre-existing cardiac disease and in those taking class I antiarrhythmics or drugs known to cause PR prolongation. ${ }^{19,20}$

\section{Disclosure}

The authors report no conflicts of interest in this work.

\section{References}

1. Hauser WA, Annegers JF, Kurland LT. Incidence of epilepsy and unprovoked seizures in Rochester, Minnesota: 1935-1984. Epilepsia. 1993;34:453-468.

2. Hauser A. Incidence and Prevalence in Epilpesy: A comprehensive textbook. Philadelphia, PA: Lippincot Raven; 1998. p. 47-57.

3. Chang B, Lowenstein D. Epilepsy. N Engl J Med. 2003;349: 1257-1266.

4. Loiseau P. Intractable epilepsy: Prognostic evaluation. In: Schmidt D, Morselli PL, editors. Intractable Epilepsy: Experimental and Clinical Aspects. New York, NY: Raven Press; 1986.

5. Doty P, Rudd G, Stoehr T, Thomas D. Lacosamide. Neurotherapeutics. 2007;4:145-148.

6. Stohr T, Kupferberg H, Stables J, et al. Lacosamide, a novel anticonvulsant drug, shows efficacy with a wide safety margin in rodent models for epilepsy. Epilepsy Res. 2007;74(2-3):147-154.

7. Perucca E, French J, Bialer M. Development of new antiepileptic drugs: challenges, incentives, and recent advances. Lancet Neurol. 2007;6:793-804.

8. Sander J. Some aspects of prognosis in the epilepsies: A review. Epilepsia. 1993;34:1007-1016.

9. Bialer M. New antiepileptic drugs currently in clinical trials: is there a strategy in their development? Ther Drug Monit. 2002; 24:85-90.

10. Löscher W, Schmidt D. Strategies in antiepileptic drug development: is rational drug design superior to random screening and structural variation? Epilepsy Res. 1994;17:95-134.

11. Cortes S, Liao Z, Watson D, Kohn H. Effect of structural modification of the hydantoin ring on anticonvulsant activity. J Med Chem. 1985;28:601-606.

12. Beyreuther B, Freitag J, Heers C, Krebsfänger N, Scharfenecker U, Stöhr T. Lacosamide: a review of preclinical properties. CNS Drug Rev. 2007;13:21-42.

13. Yoshimura T, Arimura N, Kaibuchi K. Molecular mechanisms of axon specification and neuronal disorders. Ann N Y Acad Sci. 2006;1086: $116-125$. 
14. Errington A, Stöhr T, Heers C, Lees G. The investigational anticonvulsant lacosamide selectively enhances slow inactivation of voltage-gated sodium channels. Mol Pharmacol. 2008;73: 157-169.

15. Bialer M, Johannessen S, Kupferberg H, Levy R, Perucca E, Tomson T. Progress report on new antiepileptic drugs: a summary of the Eigth Eilat Conference (EILAT VIII). Epilepsy Res. 2007;73:1-52.

16. Thomas D, Scharfenecker U, Schiltmeyer B, Doty P, Cawello W, Horstmann R. Low potential for drug-drug interaction of lacosamide. Epilepsia. 2006;47(Suppl 4):200.

17. Jatuzis D, Biton V, Ben-Menachem E, Abou-Khalil B, Doty P, Rudd G. SP667 Study Group. Evaluation of the effect of oral lacosamide on concomitant AED plasma concentrations in patients with partial seizures. Epilepsia. 2005;46(Suppl 8):170.

18. Biton V, Rosenfeld W, Whitesides J, Fountain N, Vaiciene N, Rudd G. Intravenous lacosamide as replacement for oral lacosamide in patients with partial-onset seizures. Epilepsia. 2008;49(3):418-424.

19. Ben-Menachem E, Biton V, Jatuzis D, Abou-Khalil B, Doty P, Rudd G. Efficacy and safety of oral lacosamide as adjunctive therapy in adults with partial-onset seizures. Epilepsia. 2007;48:1308-1317.

20. Halász P, Kälviäinen R, Mazurkiewicz-Beldzińska M, et al; SP755 Study Group. Adjunctive lacosamide for partial-onset seizures: Efficacy and safety results from a randomized controlled trial. Epilepsia. 2009;50:443-453.

21. Chung S, Sperling M, Biton V, Krauss G, Beaman M, Herbert D. Lacosamide: efficacy and safety as oral adjunctive treatment for partial-onset seizures (SP754) [abstract]. Epilepsia. 2007;48(Suppl 6):321.
22. Perucca E, Yasothan U, Clincke G, Kirkpatrick P. Fresh from the pipeline: lacosamide. Nat Rev Drug Discov. 2008;7:973-974.

23. Beydoun A, D'Souza J, Hebert D, Doty P. Lacosamide: pharmacology, mechanisms of action and pooled efficacy and safety data in partialonset seizures. Expert Rev Neurother. 2009;9(1):33-42.

24. Ben-Menachem E. Lacosamide: an investigational drug for adjunctive treatment of partial-onset seizures. Drugs Today. 2008;44:35-40.

25. Kellinghaus C. Lacosamide as treatment for partial epilepsy: mechanisms of action, pharmacology, effects, and safety. Ther Clin Risk Manag. 2009;5:757-766.

26. Biton V, Rosenfeld WE, Whitesides J, Fountain NB, Vaiciene N, Rudd GD. Intravenous lacosamide as replacement for oral lacosamide in patients with partial-onset seizures. Epilepsia. 2008;49:418-424.

27. Krauss G, Ben-Menachem E, Mameniskiene R, Vaiciene N, Brock M. Whitesides J. Intravenous lacosamide (SPM 927) as replacement for oral lacosamide in subjects with partial seizures; A multicenter, open-label inpatient safety and tolerability trial. Epilepsia. 2006;47(Suppl 4):163.

28. Tilz C, Resch R, Hofer T, Eggers C. Successful treatment for refractory convulsive status epilepticus by parenteral lacosamide. Epilepsia. 2009. Aug 8 [Epub ahead of print].

29. Cross SA, Curran MP. Lacosamide: in partial-onset seizures. Drugs. 2009;69:449-459.

30. Wehner T, Bauer S, Hamer HM, et al. Six months of postmarketing experience with adjunctive lacosamide in patients with pharmacoresistant focal epilepsy at a tertiary epilepsy center in Germany. Epilepsy Behav. 2009;16(3):423-425.
Neuropsychiatric Disease and Treatment

\section{Publish your work in this journal}

Neuropsychiatric Disease and Treatment is an international, peerreviewed journal of clinical therapeutics and pharmacology focusing on concise rapid reporting of clinical or pre-clinical studies on a range of neuropsychiatric and neurological disorders. This journal is indexed on PubMed Central, the 'PsycINFO' database and CAS, and is the official

\section{Dovepress}

journal of The International Neuropsychiatric Association (INA). The manuscript management system is completely online and includes a very quick and fair peer-review system, which is all easy to use. Visit http://www.dovepress.com/testimonials.php to read real quotes from published authors. 